\title{
ANÁLISIS EXPERIMENTAL DE LA CORRUPCIÓN Y DE LAS MEDIDAS ANTICORRUPCIÓN. ¿DÓNDE ESTAMOS, HACIA DÓNDE VAMOS?
}

\author{
FrÉdÉRIC BoEHM* \\ Carolina Isaza E. ${ }^{* *}$ \\ Martha Liliana Villalba Díaz ${ }^{* * *}$
}

\section{Resumen}

Este artículo presenta una discusión de la literatura interdisciplinar reciente sobre el estudio empírico y experimental de la corrupción. El análisis empírico de la corrupción se ve dificultado por su naturaleza ilícita. En las dos últimas décadas, han prevalecido estudios econométricos basados en datos de percepción de corrupción. Si bien estos han avanzado nuestro entendimiento del fenómeno, cada vez más se notan los límites de esta metodología al establecer causalidades y explorar los fundamentos de la toma de decisión corrupta o ética de los individuos. Recientemente, las literaturas económica, política, psicológica y organizacional han aplicado el método experimental para entender mejor el comportamiento corrupto y probar medidas anticorrupción. La revisión presenta y discute los principales aportes de esta literatura y así brinda un punto de partida para seguir investigando las preguntas abiertas.

Palabras clave: corrupción, experimentos, ética, comportamiento.

* Doctor en Economía. Investigador asociado, Universidad Autónoma del Caribe, Barranquilla (Colombia). frederic.boehm@yahoo.fr

** Doctora en Gobierno y Política Pública. Docente-Investigadora, Universidad Externado de Colombia, Bogotá (Colombia).carolina.isaza@uexternado.edu.co

*** Economista. Investigadora independiente. villalbam88@hotmail.com

Recibido: 28 de septiembre de 2015 / Modificado: 11 de noviembre de 2015 / Aceptado: 18 de noviembre de 2015. Para citar este artículo

Boehm, F., Isaza, C. y Villalba Díaz, M. L. (2015). Análisis experimental de la corrupción y de medidas anticorrupción. ¿Dónde estamos, a dónde vamos? OPERA, 17, pp. 105-126. DOI: http://dx.doi.org/10.18601/16578651.n17.06 
EXPERIMENTAL ANALYSIS

OF CORRUPTION AND ANTI-CORRUPTION MEASURES - WHERE ARE WE AND WHERE ARE WE GOING?

\section{Abstract}

An empirical analysis of corruption is difficult due to its illicit nature. During the last two decades, research focused mainly on econometric studies based on corruption perception data. While these studies have contributed to our understanding of the phenomenon, they face limitations with respect to the possibility of establishing causalities and understanding corrupt or ethical decision-making. This contribution reviews the economic, political, psychological and organisational literature that applies experimental methods to analyse corrupt behaviour and to test anti-corruption mechanisms. The principal results of this literature are presented and discussed in order to provide an entry point for further research.

Key words: Corruption, experiments, ethics, behaviour.

\section{INTRODUCCIÓN}

En años recientes, el enfoque experimental se ha usado de forma creciente en las ciencias sociales. En particular, la economía y la psicología han aumentado el uso de experimentos en "laboratorio" para identificar variables que inciden en el comportamiento de los individuos. También, aunque en menor medida, lo han hecho la ciencia política y otras ciencias sociales.
Este artículo aborda un ámbito innovador de aplicación de estos métodos experimentales: el estudio de la corrupción. Cuando se trata de estudiar la corrupción o el comportamiento corrupto, existen dificultades adicionales a las de observar otros comportamientos individuales; la corrupción, por su naturaleza clandestina y usualmente ilegal, rara vez se puede abordar en la investigación a través de la observación directa. La corrupción, definida como el abuso de un poder delegado para obtener beneficios privados, sesga decisiones administrativas y políticas, desvía recursos y socava el desarrollo sostenible (ver, por ejemplo, Aidt, 2011). En esa medida, es un tema de alto interés para los estudios políticos y del Gobierno; incluso se podría afirmar que el estudio de la corrupción está actualmente en el centro de las preocupaciones relacionadas con el Gobierno.

En el análisis de fenómenos sociales como este, es fundamental entender cómo toman decisiones los individuos que interactúan en una sociedad y cómo estas decisiones se ven influenciadas por el entorno, las expectativas y creencias, y la información disponible. La teoría de juegos, la psicología y la economía comportamental nos brindan herramientas para deducir predicciones teóricas sobre el comportamiento individual (Gintis, 2009; Camerer, 2003; Tversky y Kahnemann, 1986; Druckman et al., 2006). Estas predicciones nos guían en cuanto a las variables de interés para el trabajo empírico.

En este, dos tipos de observaciones son posibles. Por un lado, podemos recurrir a estudios observacionales de datos cuantitativos o cualitativos. De hecho, el análisis de la co- 
rrupción se basa a menudo en datos estadísticos, ya sea de percepción de corrupción o de victimización, es decir, reportes individuales de experiencia directa con la corrupción ${ }^{1}$.

Por otro lado, los trabajos cualitativos basados en el análisis de casos específicos o en entrevistas nos pueden ayudar a entender mejor los detalles de los mecanismos de la corrupción (por ejemplo Fink y Boehm, 2011). Sin embargo, puede resultar difícil aislar las variables e inferir relaciones causales $y$, además, los estudios de caso son difícilmente generalizables, ya que dependen fuertemente del contexto en el cual han sido observados.

Pero hay una tercera posibilidad: el estudio experimental. Este permite crear observaciones a propósito, en un entorno controlado en el cual es posible observar las variables que nos interesan y manipularlas. El estudio experimental de la corrupción es relativamente nuevo. Más que un sustituto a los trabajos cuantitativos y cualitativos, se trata de una metodología complementaria que permite arrojar luz sobre algunas preguntas difíciles de responder con aquellas.

En la literatura de economía experimental ya existen algunas revisiones de literatura sobre experimentos en corrupción (Andvig, 2005; Dusek, Ortmann y Lízal, 2005; Abbink, 2006; Bobkova y Egbert, 2012; Frank et al., 2011; Serra y Wantchekon, 2012). No existe en cambio una recopilación similar desde las otras disciplinas relevantes. Este trabajo aporta un primer esfuerzo en esa dirección y en la integración de perspectivas de diferentes disciplinas que aportan al mismo objeto de estudio con métodos experimentales.

En ese sentido, nuestro objetivo es ofrecer de manera concisa un punto de entrada para estudiosos de la corrupción en las ciencias sociales hispanoparlantes, que aún desconocen los avances logrados en los estudios del comportamiento a través del método experimental, y obtener una visión sobre los resultados ya obtenidos por este sobre la corrupción y los mecanismos anticorrupción.

Para el análisis de la corrupción, el método experimental se puede aplicar para dos tipos de preguntas. Primero, para inferir relaciones causales en el comportamiento corrupto o ético. Segundo, para observar el efecto de cambios en las reglas de juego formales. Es decir, se pueden probar mecanismos que podrían reducir riesgos de corrupción antes de implementarlos en políticas públicas a gran escala. Precisamente, tomando en cuenta el creciente desencanto con políticas anticorrupción alrededor del mundo ${ }^{2}$, un acercamiento más basado en evidencia y que establezca mecanismos causa-efecto es importante.

El artículo se divide en dos partes. En la primera, se ofrece una visión general sobre la metodología experimental que permitirá entender mejor el alcance y los límites de los resultados obtenidos hasta ahora en la literatura. Estos se presentarán en la segunda parte del artículo. A pesar de lo reciente que es el trabajo experimental sobre corrupción, ya

\footnotetext{
1 Para una discusión de estos índices de percepción de corrupción, ver por ejemplo, González y Boehm (2013).

2 Para el caso de Colombia, ver, por ejemplo, Misas (2005) e Isaza (2011).
} 
disponemos de algunos resultados robustos. El artículo concluye con una reflexión acerca del futuro del método experimental para el análisis de la corrupción en perspectiva interdisciplinar.

\section{EL MÉTODO EXPERIMENTAL EN LABORATORIOS}

Los experimentos sobre corrupción en economía empezaron a finales de los años noventa y han atraído el interés de investigadores de la corrupción que encontraban limitaciones en otros enfoques. En la psicología social y de las organizaciones, así como en la administración de empresas, los experimentos están orientados al comportamiento ético. Las preguntas sobre la ética en las organizaciones y en los grupos sociales aportan importantes elementos al estudio de la corrupción. Sin embargo, los dos enfoques tienen algunas diferencias que es importante resaltar.

\section{Fundamentos de la economía experimental}

En la economía experimental, la idea básica es crear en el laboratorio un pequeño mundo virtual (Friedman y Cassar, 2004, p. $25)^{3}$. En este interactúan dos o más individuos bajo ciertas reglas de juego establecidas por el investigador. Estas reglas de juego son exógenas y definen el conjunto de alternativas disponibles para el individuo, así como los pagos asociados. Aquí es clave distinguir entre reglas explícitas y reglas implícitas. Las reglas explícitas son definidas y controladas por el experimentador. Por ejemplo, el experimento puede consistir en analizar los efectos de diferentes reglas de subasta sobre los resultados de la misma. Las reglas implícitas, en cambio, son normas, tradiciones y hábitos informales que las personas traen consigo al laboratorio como parte de su herencia evolutiva, tanto cultural como biológica.

Además de las reglas, el experimento se define por las características de los jugadores. Nuevamente, hay características sobre las cuales el investigador tiene influencia; las que se le dan al individuo en el marco del laboratorio, y características que cada participante trae consigo, pero que también pueden ser de interés para el análisis. En particular, los individuos que participan en un experimento pueden tener preferencias diversas sobre los posibles resultados, las cuales son difíciles de conocer. Vernon Smith ideó un truco para solucionar este problema: la teoría del valor inducido. La idea es inducir, a través de incentivos adecuados, preferencias especificadas previamente, para que las preferencias innatas de los sujetos pasen al segundo plano. Para que este truco funcione, son necesarias tres condiciones (Friedman y Cassar, 2004, p. 26):

- Contingencia: los resultados del experimento deben estar conectados con un medio de recompensa (por ejemplo, dinero o barras de chocolate). Esta relación

3 En español, Rey-Biel (2008) ofrece una introducción concisa a la economía experimental; Brañas Garza (2011) permite un acercamiento profundizado. 
entre acción y resultado debe ser clara y el sujeto debe ser consciente de ella.

- Dominancia: una variación de la cantidad de recompensa recibida tiene que ser más importante para el sujeto que otros elementos de decisión. Por ejemplo, los sujetos podrían querer "complacer" o "no quedar mal” con el investigador y así no escoger la opción que les gustaría escoger en realidad. Para evitar este problema y garantizar la condición de dominancia, se suelen introducir pagos anónimos, es decir, el investigador no sabe quién recibió cuánta recompensa.

- Monotonicidad: el sujeto debe siempre preferir obtener más del incentivo y nunca llegar a estar saciado. El dinero como incentivo muy probablemente satisface esta condición, sin embargo, esto no es obvio para otros tipos de incentivos, como barras de chocolate.

Se establece una función, llamada función de pagos, que relaciona la elección dentro del conjunto de alternativas posibles con los resultados específicos del juego; en juegos con interdependencia estratégica estos pagos también dependerán de las elecciones de los otros jugadores. La teoría de juegos nos ofrece una predicción en cuanto a la elección del individuo si este se estuviera comportando de manera racional y si estuviera maximizando sus beneficios.

\section{Los experimentos desde la psicología}

La psicología social ha utilizado el método experimental desde hace décadas. De hecho, el padre de la economía experimental, Vernon Smith, se inspira en Stanford en los trabajos del psicólogo Sydney Siegel. Sin embargo, las técnicas experimentales en la economía experimental y en psicología cognitiva y social divergieron y hoy se pueden identificar cuatro diferencias metodológicas principales entre estas (Friedman y Cassar, 2004, p. 18). Primero, los economistas usan instrucciones escritas para asegurar que los sujetos entiendan lo que están jugando; en psicología este no necesariamente es el caso, aunque cada vez se hace más frecuente. Segundo, los economistas dejan que se repita el experimento varias veces y se concentran en los datos de las últimas repeticiones cuando pueden estar seguros de que los sujetos entienden bien la situación y aprendieron a jugar "bien". Tercero, los economistas siempre pagan a los sujetos de acuerdo con su desempeño, para garantizar el cumplimiento de la teoría del valor inducido. Los psicólogos, en cambio, no siempre pagan a los sujetos y, si lo hacen, suelen pagar una suma fija por participar.

Cuarto, si bien a veces no dan toda la información, los economistas nunca le mienten a los sujetos acerca de la naturaleza del experimento. En psicología, en cambio, el investigador puede engañar o mentir a los participantes. Debido al tipo de preguntas que se plantea la psicología, con frecuencia es necesario poner al participante en una situación simulada o introducir información que no es veraz para lograr que el comportamiento de interés sea observable en el laboratorio. De esta manera, se logra obtener reacciones que no se podrían observar sin ese recurso. Sin embargo, el engaño se usa cada vez menos y con mayores restricciones (Hertwig y Ortmann, 2008). 
La psicología difiere de la economía también en el instrumento principal de medición, pues usa sobre todo el cuestionario. En los cuestionarios se introducen las variables a través de preguntas y las respuestas de los participantes arrojan los diferentes valores que esas variables pueden tomar. Un segundo instrumento es la observación directa de un comportamiento, cuando se le pide a los participantes que realicen una acción, como participar en una discusión, pulsar un botón, asumir un rol, etc. En este caso, el investigador observa las alternativas de comportamiento que se presentan entre los participantes dentro de un protocolo predeterminado y estas se cuantifican o se categorizan para producir los valores. La manipulación de las condiciones del experimento se hace entonces variando lo que se pregunta o los posibles comportamientos observables durante el mismo.

En general, los métodos para asegurar el control en el diseño experimental son tres: el uso de pruebas antes y después de manipular la variable independiente, el uso de múltiples condiciones o tratamientos, y la selección y asignación aleatoria de los participantes a las condiciones. El primer mecanismo es opcional, los otros dos son obligatorios para asegurar la solidez del experimento. Hacer mediciones antes y después implica que el número de participantes en el experimento se duplica, lo cual en ocasiones no resulta práctico (Hulbert, 2004).

Igualmente, en psicología, los investigadores prestan mucha atención a la forma en la que ellos mismos se comportan durante los experimentos, las condiciones físicas en que se realizan estos, la forma de vestir, actuar y dar las instrucciones, y todos los factores del ambiente que podrían influir en la respuesta de los participantes. Esta preocupación surge de experimentos previos en que se ha comprobado que la presencia y el comportamiento del investigador y sus ayudantes tienen efectos psicológicos. Esto ha llevado a que se utilicen instrucciones escritas y en ocasiones instrucciones pregrabadas en audio y, cada vez más, experimentos conducidos en computadoras, para minimizar el efecto del investigador sobre el comportamiento de los sujetos (Hulbert, 2004).

\section{Ventajas y limitantes del método experimental en laboratorios}

La ventaja principal del método experimental en el laboratorio es que el investigador tiene un control directo sobre muchas variables. El arte reside en traducir la situación de interés a una versión simplificada, para estudiarla en el laboratorio. Un experimento bien diseñado permitirá hacer inferencias causales a través de la manipulación exógena, por parte del investigador, de algunas variables de interés. Siguiendo la definición de Guala (2002, p. 262), un experimento es internamente válido cuando el investigador puede atribuir la producción de un efecto $\mathrm{B}$ a un factor (o varios factores) A, y A es realmente la causa de B en el ámbito del diseño experimental E.

Sin embargo, a menudo surge la duda de hasta qué punto lo que se observó en el laboratorio bajo el diseño experimental pueda extrapolarse a cómo se comportarían los individuos en el mundo real o en otros diseños experimentales. Aquí, se critica la validez 
externa de los experimentos ${ }^{4}$. En particular, siguiendo a Armantier y Boly (2012), existen cinco puntos que pueden hacer que un experimento difiera de manera significativa del mundo real. Primero, lo que está en juego puede ser muy diferente; en un experimento el sujeto puede perder algo de dinero, pero no le está pasando nada grave realmente. Segundo, el juego puede diferir de la situación real si no se captaron todos los aspectos importantes de la misma. La simplificación, que permite obtener la validez interna, puede entonces crear resultados artificiales que no permitirían sacar conclusiones sobre el mundo real. Tercero, los jugadores pueden ser diferentes. En el laboratorio, normalmente, se recurre a estudiantes y se asignan los roles de manera aleatoria. Por un lado, el "rol" en el mundo real puede ser endógeno y, por ende, no aleatorio; por ejemplo, cuando cierto tipo de personas escoge ser gerente o funcionario. Por otro lado, autores como Stoker (2010) critican el uso de estudiantes para investigar la toma de decisiones por parte de personas altamente formadas y especializadas en el mundo real. Cuarto, los sujetos en el laboratorio saben que están siendo analizados, y esto puede influenciar su comportamiento. Quinto, muchos experimentos en laboratorios suelen llevarse a cabo en países desarrollados, lo que tiene un impacto sobre los sujetos escogidos y podría dificultar la generalización a países en desarrollo.
Además, otro motivo de cautela ya mencionado se refiere a la influencia del investigador en el experimento. Si el experimento no es conducido con la máxima atención a los detalles, es posible que los resultados se vean afectados por el lenguaje verbal y no verbal (framing) del experimentador y esto los invalide. Algunos de estos puntos se pueden mitigar a través de un buen diseño, sin embargo, quedarán siempre dudas en cuanto a la validez externa.

Una respuesta parcial a estas dudas sobre la validez externa son los experimentos de campo, que consisten en asignar en el mundo real unidades de análisis (individuos, escuelas o municipios, por ejemplo), ya sea a un grupo de tratamiento o a un grupo de control (Serra y Wantchekon, 2012, cap. 1) $)^{5}$. Los experimentos de campo difieren de los experimentos en un laboratorio en que los sujetos, en su ambiente natural, no saben que están participando en un experimento. Lo que se gana del lado de la validez externa, se pierde, sin embargo, por el lado de la validez interna y de la posibilidad de generalizar los resultados a otros entornos.

Así, es mejor considerar los experimentos en laboratorios y en el campo como metodologías complementarias. De todas formas, Armantier y Boly (2010) presentan un resultado reconfortante. Hicieron el mismo experimento sobre corrupción en tres ambientes diferentes: en un laboratorio en un país desa-

\footnotetext{
4 Una discusión detallada sobre la validez externa se encuentra en Moro y Freidin (2012).

5 Se habla de un "experimento natural", cuando el investigador no tiene control sobre el aspecto aleatorio (Dunning, 2012).
} 
rrollado, en un laboratorio en un país en vía de desarrollo y en un experimento de campo en un país en vía de desarrollo. No encontraron diferencias estadísticamente significativas entre los tres, lo cual valida el uso del laboratorio y además estaría apuntando a que sí existen algunas características de la corrupción que son universalmente válidas.

En las dos siguientes secciones presentamos los resultados más destacados de la literatura multidisciplinaria. El foco de esta revisión está en los resultados, sin entrar en los detalles de los diseńos experimentales; para estos, invitamos a los lectores a ver las fuentes originales.

\section{EXPERIMENTOS QUE MEJORAN NUESTRO} ENTENDIMIENTO SOBRE LA CORRUPCIÓN

Se indican a continuación los resultados principales de los experimentos sobre corrupción, organizados desde las variables de interés que han sido probadas por diferentes autores.

\section{Reciprocidad}

En primer lugar, los estudios experimentales demuestran claramente la importancia del rol de la reciprocidad (Lambsdorff, 2012). Las normas de reciprocidad y los niveles de confianza mutua conducen a relaciones estables de intercambio, reduciendo los costos de transacción, sobre todo los costos del con- trol. Dado que la corrupción es un acuerdo informal, sin respaldo legal, las instituciones informales como la confianza o las normas de reciprocidad juegan un factor clave en una relación corrupta para evitar el oportunismo ${ }^{6}$. De hecho, los que sobornan no pueden estar seguros de si serán retribuidos o no, pero esperan una actitud recíproca. En caso de que no se cumpla con el acuerdo, puede jugar un papel el nivel de reciprocidad negativa, es decir la voluntad de sancionar o denunciar la parte oportunista del acuerdo.

Los experimentos muestran que las relaciones que se repiten favorecen el establecimiento de confianza y reciprocidad. En el experimento diseñado por Abbink et al. (2002), los autores demuestran que relaciones repetitivas permiten la emergencia de normas de reciprocidad corrupta entre los jugadores y que estas relaciones cooperativas y corruptas se afiancen con el tiempo.

Por otro lado, el monto del soborno podría ser un incentivo para la reciprocidad positiva: entre más alto el valor, más fuerte el sentimiento de tener que retribuir y cumplir con el favor corrupto. Esto precisamente es lo que observan Armantier y Boly (2011) en un experimento de campo realizado en Burkina Faso. Los autores observan que al doblar el monto de los sobornos aumentaron tanto la tasa de aceptación de estos como la probabilidad de que los receptores del soborno sean recíprocos.

\footnotetext{
6 Para una discusión no experimental de estos costos de transacción e instituciones ideadas por los actores corruptos para evitar el oportunismo, ver Boehm y Lambsdorff (2009).
} 


\section{Costo social de la corrupción}

Otro tema de interés es el costo social de la corrupción, por lo que cabe preguntarse si los corruptos tienen conciencia del daño que generan a la sociedad y si esto afecta sus decisiones. Como respuesta a esto, Abbink et al. (2002) encontraron que el ser consciente de la externalidad negativa que genera la corrupción y saber a quién afecta no tiene efectos sobre la decisión de ofrecer o aceptar un soborno. Dado este resultado, queda una duda acerca de la efectividad que puedan tener las campañas que resaltan los costos de la corrupción, una medida común en la práctica, por lo que este dinero se podría invertir de manera más eficiente (Dusek et al., 2005). Este resultado desalentador se confirma en Büchner et al. (2008), quienes encuentran que ser consciente de que la corrupción es perjudicial para la sociedad no ayuda.

En cambio, en un experimento diferente, que se enfoca en la pequeña corrupción, y no en un soborno entre empresario y funcionario público como los estudios anteriores, Barr y Serra (2009) sí encuentran que saber del costo impuesto a terceros reduce la tendencia del ciudadano a ofrecer sobornos. Así, campañas especialmente diseñadas para mostrar el costo social de la pequeña corrupción podrían tener un efecto, mientras que campañas contra la corrupción de alto nivel no lo tendrían.

\section{Características socioeconómicas de los implicados}

Un aporte importante de los estudios experimentales es que nos ayudan a entender mejor a los actores de un acuerdo corrupto, controlando por características socioeconómicas de los sujetos. En particular, llama la atención que la diferencia de género en el comportamiento corrupto es notoria (Frank et al., 2009). En un trabajo de Alatas et al. (2009), los autores analizaron el comportamiento de hombres y mujeres en cuatro países (India, Singapur Indonesia, Australia). Encontraron que los hombres se comportan de manera bastante similar, independientemente de su país de origen, ofreciendo sobornos con mayor frecuencia y montos más altos que las mujeres. En cambio, el comportamiento de las mujeres difiere de manera significativa dependiendo de su origen. Posiblemente, esto muestra que existe mayor variación en el rol social de las mujeres que en el de los hombres. Lambsdorff y Frank (2011), a su vez, demuestran que las mujeres son más propensas a reportar un soborno que los hombres. Adicionalmente, encuentran que las mujeres tienden a tener una actitud más oportunista que los hombres, es decir, aceptan el soborno, pero no son recíprocas con el favor corrupto esperado.

Por otra parte, Armantier y Boly (2011), en un experimento de campo, observaron que la probabilidad de aceptar un soborno se reduce con la edad, la religiosidad y la capacidad de hacer bien su trabajo. A su vez, Frank y Schulze (2000) encontraron que el comportamiento difiere por la carrera profesional de los individuos. En su experimento analizan la diferencia entre los economistas y estudiantes de otras carreras, observando que los economistas son más corruptibles. Los autores encuentran que la razón de este comportamiento no se debe a un efecto de aprendizaje o adoctrinamiento 
durante la carrera, sino a una autoselección: los que deciden estudiar economía son más vulnerables.

\section{Incertidumbre y riesgo}

La incertidumbre del éxito o el fracaso de un soborno también afecta la decisión de ofrecerlo o aceptarlo, y varía dependiendo del nivel esperado de reciprocidad, de la vigilancia y de la percepción que los individuos tengan del control. Es así como un aumento del nivel de incertidumbre podría reducir la corrupción. La evidencia experimental sugiere que las creencias de los individuos sobre un posible castigo tienen una influencia sustancial en el comportamiento corrupto. Por esto, se recomienda que se gradúe el nivel de incertidumbre de las creencias individuales sobre posibles auditorías aleatorias y castigos (Berninghaus et al., 2013). En la próxima sección presentaremos experimentos que resaltan los efectos del control y de las sanciones.

\section{Efectos de marco o presentación del experimento}

Algunos experimentos analizaron si existen efectos de framing, es decir, si la manera como se presenta la situación corrupta y las palabras usadas, tiene una influencia sobre el comportamiento de los sujetos o no. Dada la connotación peyorativa e ilegal de la corrupción, los sujetos podrían reaccionar de manera adversa si la oferta corrupta es demasiado directa. Los experimentos parecen demostrar que en el momento de solicitar u ofrecer un soborno, la forma en que se hace tiene gran in- fluencia en la decisión de aceptar o no. Llamar a un pago de manera explícita "soborno" y no de manera más neutral una "oferta", y hablar de jugador A y B y no de funcionario y ciudadano, tiene como efecto que se soliciten y ofrezcan menos sobornos (Barr y Serra, 2009).

Sin embargo, Lambsdorff y Frank (2010) encuentran que llamar a un pago de manera explícita un "soborno" en vez de un "regalo" aumenta la probabilidad de éxito del soborno, ya que el uso de la palabra "soborno" implica una noción de reciprocidad y una amenaza de castigo en caso de oportunismo y, así, presiona al comportamiento recíproco por parte del sobornado.

\section{Características psicológicas de los implicados}

En otro ámbito, los experimentos de psicología han mostrado que las características psicológicas individuales tienen una incidencia importante en los comportamientos no éticos. Por ejemplo, la identidad moral de una persona influye en qué tanto sus acciones éticas se verán afectadas por el poder; es decir, si el poder corrompe o no (DeCelles et al., 2012).

Asimismo, el experimento de Beu et al. (2003) mostró que hay varias características individuales que inciden en la intención de actuar de forma ética o no ética. Los autores encuentran que entre mayor sea el nivel de desarrollo moral de una persona, de acuerdo con las medidas estandarizadas usadas en psicología desde Kohlberg, mayor es la probabilidad de comportarse éticamente. El locus de control interno se relaciona también con la intención 
ética; es decir, personas con tendencia a situar las normas interna y no externamente, son más éticas que quienes actúan solo por miedo al castigo externo. Los autores también observaron que la personalidad maquiavélica era el mayor predictor de las intenciones no éticas. La agresividad, la hostilidad y el género (masculino) fueron otras características relevantes para predecir corrupción.

Por otra parte, el autocontrol de las personas es uno de los elementos que les permiten permanecer honestas incluso frente a incentivos para ser corruptas. Sin embargo, diferentes experimentos psicológicos muestran que al autocontrol es un recurso que se desgasta con el uso y que, a mayor desgaste del autocontrol, mayor probabilidad de que las personas sean deshonestas. Esto se explica introduciendo otro factor en los experimentos, que es la conciencia moral o el reconocimiento de la naturaleza ética de una situación, la cual se demostró que disminuye cuando el autocontrol está agotado y resultó ser el factor que media entre esta situación y el comportamiento no ético (Gino et al., 2011).

\section{Influencia de otros actores a través del ejemplo}

También, el ejemplo de otras personas comportándose de forma deshonesta puede incidir sobre el comportamiento de los demás, en dos sentidos. Una serie de experimentos muestra que si se trata de un miembro del mismo grupo, verlo comportarse de forma deshonesta genera un ejemplo que es seguido por los demás; si se trata de un miembro de otro grupo rival, el efecto es el contrario y los participantes se comportan de forma más honesta que en el grupo de control (Gino et al., 2009).

En la misma dirección, Gino y Bazerman (2009) muestran en el laboratorio que la erosión del comportamiento ético en otros es aceptable para el grupo cuando ocurre de manera gradual más que cuando ocurre repentinamente. Los resultados muestran que los participantes no perciben la variación gradual en las acciones y que esta percepción errada no se explica por factores conscientes o deliberados, lo cual se mide con pruebas de control y con la medición de los tiempos de respuesta. La explicación propuesta para este fenómeno es la existencia de un sesgo implícito que impide al observador ver el comportamiento como no ético, en la medida en que se desarrolla lentamente en el tiempo. Esto quiere decir que no necesariamente se trata de una decisión consciente de ignorar la corrupción.

Esto sugiere que una organización puede deslizarse hacia la corrupción sin que nadie se dé cuenta de lo que está ocurriendo y, por tanto, sin que nadie lo denuncie. También sugiere que es preferible reaccionar ante la corrupción desde los hechos más pequeños y aparentemente inanes, pues estos pueden ser el inicio de un camino hacia comportamientos más graves generalizadamente aceptados y se podría generar un círculo vicioso manteniendo la organización en un equilibrio corrupto.

\section{Factores organizacionales e incentivos}

En cuanto a los factores organizacionales, la cultura de la organización es importante en la medida en que un ambiente que resalte 
o espere personas egoístas que maximizan el beneficio individual aumenta los comportamientos no éticos (Burke, 2009). Esto puede explicar que, como se mencionó, en muchos experimentos los estudiantes de economía se comporten de forma menos altruista que los estudiantes de otras carreras. Esto podría deberse a que en la formación en economía se les refuerzan marcos de referencia de un individuo egoísta y autointeresado (Frank et al., 1996), o bien por un proceso de autoselección de los estudiantes, como lo parece sugerir el experimento de Frank y Schulze (2000) citado arriba.

\section{Motivaciones intrínsecas y autoimagen}

En definitiva, algunos de los experimentos desde la psicología y la economía proponen que los incentivos externos, tales como el castigo, no son los únicos factores que inciden en el comportamiento ético. Mazar y Ariely (2006) afirman:

In contrast to this classic economic perspective, there exists ample evidence from different academic fields such as psychology, sociology, anthropology, and behavioral and experimental economics, as well as neuroeconomics and neuroscience, that in addition to the external reward mechanisms, there also exist internal reward mechanisms and that these exert influence on individuals' decisions.

Ejemplos de estos mecanismos internos son el mantenimiento de una autoimagen de persona honesta, justa o altruista. En investigaciones con imágenes neurológicas se ha visto que las mismas zonas del cerebro se activan para las recompensas externas (premios) e in- ternas (aprobación social y satisfacción moral) (Mazar y Ariely, 2006).

Por otra parte, la capacidad de justificación y autoengaño explica en gran medida por qué las personas logran ser deshonestas sin dańar su autoimagen y, por tanto, sin sentirse culpables. De acuerdo con los extensos experimentos que han realizado Ariely et al., la mayoría de las personas en diversas sociedades engañan o mienten por beneficio propio solo hasta cierto límite, en el cual se activan los mecanismos internos y se evita una deshonestidad que iría en contra de su autoimagen positiva. Una vez estos se activan, la persona es insensible a los incentivos externos, pues la motivación interna prima. Sin embargo, ese pequeño margen en el que se puede ser deshonesto sin sufrir en la autoimagen puede variar según las estrategias de justificación, racionalización y autoengaño que use cada persona (Mazar y Ariely, 2006). De hecho, en otro experimento se vio que las personas que son más creativas logran engañarse a sí mismas con argumentos más convincentes y, por tanto, pueden ser más deshonestas (Gino y Ariely, 2012).

Otra forma en la que se facilitan los actos corruptos sin sentir culpabilidad directa, y que además ayuda a reducir la incertidumbre de éxito o fracaso en un intento de establecer una relación corrupta, es la introducción de los intermediarios en las transacciones. Estos logran disminuir considerablemente la incertidumbre y causan un aumento significativo en la corrupción, eliminando además los costos morales del acto corrupto (Drugov et al., 2014). 
EXPERIMENTOS QUE MEJORAN NUESTRO ENTENDIMIENTO SOBRE MEDIDAS ANTICORRUPCIÓN

Los experimentos que nos ayudan a entender mejor los determinantes y los actores de la corrupción presentados en la sección anterior tienen implicaciones políticas. Sin embargo, se pueden llevar al laboratorio o estudiar en el campo medidas anticorrupción tradicionales, como sanciones, controles, salarios más altos, transparencia o competencia, así como medidas innovadoras que no se utilizan actualmente. En algunos casos, las medidas sugeridas por los experimentos son muy simples y de bajo costo, como se verá.

\section{Pureza simbólica}

Para empezar, algunos experimentos han identificado acciones simbólicas que ayudan a incentivar el comportamiento ético, en particular acciones relacionadas con la limpieza física. Zhong y Liljenquist (2006) encuentran que una situación referida a un comportamiento no ético despierta en las personas el deseo de limpieza física y asociaciones con conceptos referidos al aseo. Esto significa que los comportamientos no éticos, psicológicamente pueden ser "limpiados" con actos físicos de purificación, lo cual ha sido denominado "efecto Macbeth". Este sería también el caso del olor a limpio que se mencionó antes y de las condiciones de iluminación utilizadas durante algunos experimentos sobre comportamiento ético. Cuando la luz es mejor, el comportamiento deshonesto disminuye. Incluso en la presencia de espejos (Heine et al., 2008) o de un dibujo de ojos (Bateson et al., 2006) las personas suelen comportarse de forma más honesta por el efecto de sentirse observadas, aunque esto varía entre diferentes culturas. Todos estos factores remiten a las asociaciones culturales, basadas en reacciones neurológicas, entre el asco y el rechazo hacia comportamientos no éticos.

En términos de medidas anticorrupción, estos curiosos resultados indican que crear ambientes limpios e iluminados puede inhibir al menos hasta cierto punto el comportamiento corrupto. Incluso, se ha demostrado que la presencia de un olor asociado con "limpieza" lleva a aumentar el comportamiento prosocial de las personas (Liljenquist et al., 2010). Esto podría utilizarse en las organizaciones públicas y en los lugares en los que podría ocurrir la corrupción, así como el crear la sensación de transparencia física, con ventanas y vidrios que permitan ver a los trabajadores o a los políticos.

\section{Resaltar la dimensión ética de una situación}

En un segundo sentido, los experimentos sugieren medidas relacionadas con destacar el carácter ético de una decisión. Es decir, cuando las personas actúan y toman decisiones, no siempre son conscientes de las dimensiones éticas de la misma, de forma que recordarles o hacerles visible la connotación ética puede hacer una gran diferencia en su comportamiento. Dan Ariely et al., demostraron esto utilizando declaraciones firmadas al inicio de una prueba, que invocan el código de honor o de ética de la organización o, incluso, los diez 
mandamientos. En los experimentos, estas declaraciones firmadas redujeron en $100 \%$ el comportamiento deshonesto, incluso en la ausencia total de vigilancia y castigo (Mazar et al., 2008; Mazar y Ariely, 2006). Este tipo de medidas pueden ayudar también a activar los incentivos internos relacionados con la autoimagen, al hacer conscientes a las personas de que comportarse de cierta manera sería no ético o corrupto e invocar así su deseo de verse como una "buena persona” frente a sí mismo $\mathrm{y}$ frente a otros.

\section{Gestión de conflictos de interés}

Otra recomendación relevante desde esta literatura se relaciona con los conflictos de interés y las formas de prevenirlos. Se hicieron experimentos en que se mostró que las políticas de publicitar dichos conflictos no son adecuadas, pues las personas no descuentan en la medida suficiente el conflicto de interés a la hora de seguir los consejos autointeresados de quienes están afectados por el mismo. Incluso sabiendo que alguien tiene un conflicto de interés y que va a asesorar mal una decisión, su opinión se toma en cuenta y la decisión resulta sesgada y en contra del interés general (Ariely, 2012). Esto lleva a pensar que se debería excluir del todo de las decisiones a personas que tengan un conflicto de interés en estas.

\section{Sanciones}

En relación con los incentivos externos, hay evidencias contradictorias que señalan que en algunas condiciones estos se activan y en otras priman los incentivos internos o la motivación intrínseca descritos arriba. Abbink et al. (2002) observan que las sanciones exógenas afectan negativamente los sobornos, aun cuando la probabilidad de detección es baja, por lo que concluyen que la sanción actúa como un disuasivo efectivo. Además, encuentran que los individuos tienen la tendencia a sobrestimar la probabilidad de ser detectado y castigado. A su vez, Büchner et al. (2008) encuentran que a las personas no les importa participar en un soborno cuando no hay una amenaza de una sanción legal, lo que muestra de nuevo la importancia de la imposición de castigos o sanciones.

Sin embargo, algunas sanciones, lejos de persuadir de los actos corruptos, los incentivan, al reemplazar la motivación intrínseca por la extrínseca (Abbink y Serra, 2012). Campos Ortiz (2011) encuentra que en un ambiente corrupto, si las políticas anticorrupción son muy agresivas, por ejemplo, con aumentos de penas fuertes, los corruptos suelen simplemente pedir sobornos más altos. Esto sugiere que la decisión de ser corrupto incluye un cálculo de riesgos y ganancias.

\section{Vigilancia y control}

En cuanto a los efectos del control, Schulze y Frank (2003) muestran que este puede tener un efecto negativo sobre los agentes, al disminuir su honestidad a través de la destrucción de su motivación intrínseca. Los autores observan que en un tratamiento sin riesgos había más sujetos perfectamente honestos que en un tratamiento con riesgo de detección. Esto es consistente con las conclusiones de los estudios de psicología citados en la sec- 
ción anterior. En su experimento de campo, Armantier y Boly (2011) confirman la validez externa de estos resultados: al implementar un monitoreo de bajo nivel, la medida es efectiva, pero si se intensifica el monitoreo, se desplaza la honestidad o lealtad del individuo hacia su jefe si se siente demasiado controlado. Pero por supuesto, el control sigue siendo importante. Así, Lambsdorff (2012) sugiere separar las funciones de control de las del superior jerárquico, para que el control no dañe la relación de lealtad positiva.

Aparte de esto, el control puede ser oficial, desde entidades de control interno y auditoría externa, o se puede tratar de un control social ejercido por la sociedad civil. Con respecto al control social, los resultados de los experimentos son contradictorios. Por una parte, Olken (2007), en un experimento de campo, encuentra que la participación ciudadana no es efectiva para disminuir la corrupción, mientras que las auditorías oficiales sí lo son. Por otra parte, Bertin (2010) diseñó un juego en el cual un sujeto en el rol del ciudadano puede castigar a sujetos en el rol de actores que cometen un acto corrupto. El autor observa que el ciudadano castiga al funcionario público cuando este se comporta de manera no ética, aunque esto implique para él un costo. Sin embargo, el autor observa que el ciudadano es más propenso a castigar la distribución inequitativa de los fondos que la aceptación de un soborno per se, y concluye que el castigo altruista es complejo y que aún falta investigar mucho sobre este tema.

La diferencia entre los dos trabajos se debe posiblemente a que en el caso de Bertin (2010), el ciudadano tenía la opción de casti- gar y definir la magnitud del castigo, mientras que en Olken (2007), los ciudadanos pueden auditar a los funcionarios y son invitados a participar en la rendición de cuentas, pero no tienen la opción de sancionar directamente.

\section{Salario}

Otra variable sugerida por la teoría es el nivel del salario. Este podría influir en la toma de decisión corrupta, ya que constituye un costo de oportunidad que se agrega a la sanción oficial: si llega a ser detectado, el individuo pierde su trabajo y su salario; entre más alto su salario, más alta la pérdida (Campos Ortiz, 2011). Schulze y Frank (2003), en su experimento sin riesgo, observan que el salario no tiene efecto, pero al introducir el riesgo de ser descubierto, sí hay un costo de oportunidad y un salario fijo disminuye los niveles de sobornos. Veldhuizen (2013) confirma este resultado. Aumentar el salario reduce de manera significativa la corrupción, pero sus resultados sugieren que este efecto positivo depende de la existencia de controles. En su experimento de campo, Armantier y Boly (2011) encuentran que un salario alto disminuye la probabilidad de aceptar un soborno.

Azfar y Nelson (2007), en un experimento que simula una elección presidencial (con oportunidad de reelección) auditada por un fiscal y con riesgo de ser expuesto ante la ciudadanía si el presidente roba, observan que en las sesiones de alto salario los participantes roban menos. Con salario alto, el presidente electo tiene incentivos de volver al poder y ser honesto, ya que los votantes castigan al presidente que haya sido corrupto no reeligiéndo- 
lo. Este experimento confirma que el salario representa un costo de oportunidad cuando existe una probabilidad de ser descubierto y sancionado.

\section{Transparencia}

Otro mecanismo anticorrupción establecido es la transparencia. La transparencia se puede observar en experimentos al variar el nivel de información que se transmite a los sujetos. Por ejemplo, Azfar y Nelson (2007) observan que los individuos rara vez reeligen a un político que haya sido expuesto como corrupto $^{7}$. El experimento confirma que la transparencia juega un papel en la lucha contra la corrupción, pero la evidencia también muestra que no logra eliminar los sobornos, más bien conduce solo a que roben menos para evitar ser descubiertos.

Por su parte, Peisakhin y Pinto (2010), en un experimento de campo en la India, investigaron el efecto de una ley de acceso a la información. En los slums de New Delhi es común que los funcionarios pidan sobornos para proveer bienes y servicios públicos a las personas de bajos recursos. En el experimento, observan que la nueva ley aumentó la transparencia en el gobierno local. En vez de pagar un soborno a un funcionario público, los ciudadanos pueden hacer uso del derecho de acceso a la información para obtener los bienes o servicios públicos. Los autores encontraron que si bien los ciudadanos que optan por sobornar para adquirir el bien público lo obtienen más rápido, los que eligen usar su derecho al acceso a información también tienen resultados efectivos.

En un experimento con estudiantes de secundaria, De Fine Licht et al. (2014) muestran que diferentes tipos de transparencia tienen un impacto diferente sobre la legitimidad percibida de un proceso de decisión. Los estudiantes leen diferentes escenarios sobre dos decisiones tomadas por su conferencia escolar. En el tratamiento sin transparencia solo se notifica la decisión sobre los dos temas. Los tratamientos con transparencia se dividen en dos: transparencia acerca de la racionalidad de la decisión y transparencia procesal. El primero incluía en la descripción justificaciones (argumentos) acerca de por qué se tomaron las decisiones específicas. Para el tratamiento de transparencia procesal se usaron tres estrategias: 1) se notifica la existencia de una camera web que filma en tiempo real las discusiones en la conferencia (los participantes pueden hacer uso o no de esta herramienta); 2) se da un informe parcial de las discusiones en la conferencia con un enfoque que refleja una interacción de negociación; 3) se da un informe parcial que refleja una interacción deliberativa (intercambio de argumentos). Luego de leer el escenario que les correspondía, los participantes del experimento respondieron a una serie de preguntas que recogían su opinión sobre la toma de decisión. Los resultados muestran de manera significativa que entre

\footnotetext{
7 Sin embargo, la evidencia de la ciencia política sobre accountability electoral muestra que en la práctica los políticos corruptos son reelegidos con mucha frecuencia.
} 
más transparente el proceso de toma de decisión y entre más aprenden los participantes sobre las razones de la decisión, más alta es la percepción de legitimidad de los participantes sobre el proceso. El mejor resultado se obtiene a través de la transparencia "deliberativa". En cambio, la posibilidad de observar en tiempo real la toma de decisión no es significativamente mejor que la transparencia enfocada en la racionalidad de la toma de decisión.

\section{Competencia}

Finalmente, cabe resaltar el rol potencial de la competencia, la cual puede reducir el poder monopolístico de los agentes y su posibilidad de aprovechar su poder de forma corrupta. Ryvkin y Serra (2013) prueban la efectividad del aumento en la competencia entre los funcionarios que proveen el mismo bien o servicio como política anticorrupción. Los autores encuentran que aumentar el número de funcionarios solo reduce los sobornos si esta medida al mismo tiempo reduce los costos de búsqueda para el cliente. De lo contrario, un aumento en la competencia no tiene efectos o tiene un efecto positivo sobre la demanda de sobornos por parte de los funcionarios, dependiendo de la magnitud de los costos de búsqueda.

Otra medida que se propone para romper el monopolio del funcionario público y dificultar la corrupción es implementar el "principio cuatro ojos" que consiste en que se necesite más de una firma para la toma de decisiones. La idea básica es que debería ser más difícil sobornar a dos personas que a una. Schikora (2010) diseña un experimento en el cual dos personas tienen que ponerse de acuerdo para aceptar una oferta de soborno. Observa que en estos casos hay más sobornos. El autor relaciona este resultado con otros experimentos de la literatura que demuestran que los grupos son más egoístas y logran cultivar mejor una reputación de reciprocidad.

\section{¿A DÓNDE VAMOS?}

El enfoque actual de prevención de la corrupción se basa en gran medida en el modelo tradicional de la elección racional que consiste en describir la toma de decisión de los individuos desde la maximización de sus intereses a través de un análisis de costobeneficio. Así, las recomendaciones estándares hacen explícitamente hincapié en aumentar los costos y reducir los beneficios esperados de la corrupción, por ejemplo, a través de medidas de control y de sanciones. Además, buscan reducir la discrecionalidad de los tomadores de decisiones y gestionar los riesgos de corrupción que surgen a través regulaciones de conflicto de interés o proporcionando orientación para la conducta esperada a través de códigos de ética o de conducta. Sin embargo, se nota una creciente preocupación con respecto a la falta de eficacia de estas medidas tradicionales, las cuales a veces incluso parecen tener efectos indeseados (Anechiarico y Jacobs, 1996).

Al mismo tiempo, la investigación teórica y empírica en varias disciplinas de las ciencias sociales ha ayudado al avance de nuestra comprensión de la toma de decisiones real de los individuos. En particular, la investigación experimental revisada aquí pone de relieve 
la importancia de factores como la motivación intrínseca, el orgullo, la confianza y la reciprocidad tanto negativa como positiva, $y$ ha profundizado nuestra comprensión de la corrupción. Además, el método experimental ofrece la posibilidad de probar nuevos mecanismos anticorrupción a pequeńa escala y de manera rigurosa, antes de implementarlos a través de políticas costosas en el mundo real.

Nuestra revisión de literatura evidencia algunos resultados sorprendentes. La evidencia experimental nos muestra que la mayoría de las personas no son corruptas, que la corrupción puede resultar de las condiciones específicas de una situación y no de algo inherentemente deshonesto. Sin embargo, también está claro que algunas características individuales pueden impulsar a las personas a escoger opciones no éticas ante una situación que los beneficie. Esta combinación entre características y circunstancias nos ayuda a entender por qué ha sido tan difícil reducir los niveles de corrupción en todo tipo de organizaciones y culturas.

Adicionalmente, las personas parecen tener, en general, un deseo fuerte de ser percibidas como honestas y de mantener su propia imagen de personas éticas; esto tiene que ayudar en la construcción de medidas que no asuman una naturaleza malvada o corrupta en las personas, sino, por el contrario, que fomenten la confianza y que permitan señalar cuándo una acción tiene un carácter corrupto y evitar que la gente la tome, resaltando el dańo a su autoimagen que esto implicaría.

Muchos de los resultados presentados en las secciones anteriores tienen implicaciones políticas claras. Estas ideas comienzan a encontrar su camino en el debate político internacional. El informe más reciente sobre el desarrollo del Banco Mundial, pone de relieve la importancia de la investigación de la mente y de la conducta humana, y contiene, entre otras, una sección dedicada a la corrupción (Banco Mundial, 2015). En el Reino Unido, el Gobierno conformó la Behavioural Insights Unit, ahora independiente del Gobierno, para establecer el puente entre la investigación y la política pública y aplicar soluciones novedosas a los problemas de la sociedad ${ }^{8}$. La unidad trabajó, por ejemplo, sobre el tema del fraude. Recientemente, una orden ejecutiva de septiembre 2015 del gobierno federal de Estados Unidos también busca fomentar el uso de políticas públicas basadas en la economía y psicología comportamental? .

Sin embargo, también hay un conjunto de evidencias contradictorias que sugieren áreas para la investigación futura. Todavía no es claro, por ejemplo, el papel preciso que juegan los incentivos externos, como el castigo y el control. Aún debemos identificar las condiciones en las cuales los incentivos externos se activan y aquellas en las cuales los incentivos internos y las motivaciones intrínsecas son más poderosos, para poder

\footnotetext{
8 http://www.behaviouralinsights.co.uk/

9 https://www.whitehouse.gov/the-press-office/2015/09/15/executive-order-using-behavioral-science-insightsbetter-serve-american
} 
diseñar medidas diferenciadas. Se necesita más tiempo para aclarar los resultados contradictorios e identificar las medidas que pueden ser exitosas para crear ambientes de mayor integridad, tanto en el sector público como en el privado. La psicología y la economía del comportamiento son hoy en día las áreas más activas y prometedoras para seguir avanzando en entender la corrupción y diseñar mejores políticas de prevención.

\section{REFERENCIAS}

Aвbink, K. (2006). Laboratory experiments on corruption. En Rose-Ackerman, S. (ed.). International Handbook on the Economics of Corruption (pp. 418-440). Cheltenham: Edward Elgar.

Abbink, K.; Irlenbusch, B. y Renner, E. 2002. An Experimental Bribery Game. Journal of Law, Economics, and Organization, 18 (2), 428-454.

Abbink, K. y Serra, D. (2012). Anti-corruption Policies: Lessons from the Lab. En WantcheKon, D. y Wantchekon, L. (eds.) Advances in Experimental Research on Corruption. London: Emerald Books.

Aidt, T. S. (2011). Corruption and Sustainable Development. En Rose-Ackerman, S. y Søreide, T. (eds.). International Handbook on the Economics of Corruption (pp. 3-51). Cheltenham: Edward Elgar.

Alatas, V.; Cameron, L.; Chaudhuri, A.; Erkal, N. y Gangadharan, L. (2009). Subject Pool Effects in a Corruption Experiment: A Comparison of Indonesian Public Servants and Indonesian Students. Experimental Economics, 12 (1), 113-132.

Andvig, J. C. (2005). Experimental economics and corruption: a survey of budding research. En Transparency International (ed.). Global Co- rruption Report. Corruption in Construction and Post-conflict Reconstruction (pp. 265-267). London: Pluto Press.

Anechiarico, F. y Jacobs, J. B. (1996). The Pursuit of Absolute Integrity. How corruption control makes government ineffective. Chicago: University of Chicago Press.

Armantier, O. y Boly, A. (2010). Can Corruption Be Studied in the Lab? Working Paper.

Armantier, O. y Boly, A. (2011). A controlled field experiment on corruption. European Economic Review, 55, 1072-1082.

Armantier, O. y Boly, A. (2012). On the external validity of labroatory experiments on corruption. En Wantchekon, D. y Wantchekon, L. (eds.). Advances in Experimental Research on Corruption. London: Emerald Books.

Ariely, D. (2012). The Honest Truth about Dishonesty: How We Lie to Everyone - Especially Ourselves. New York: Harper Collins.

Azfar, O. y Nelson, W. R. Jr. (2007). Transparency, Wages, and the Separation of Powers: An Experimental Analysis of Corruption. Public Choice, 130 (3/4), 471-493.

Banco Mundial (2015). World Development Report 2015: Mind, Society, and Behavior. Washington y New York: International Bank for Reconstruction and Development / The World Bank.

Bateson, M.; Nettle, D. y Roberts, G. (2006). Cues of being watched enhance cooperation in a realworld setting. Biology Letters, 2 (3), 412-414.

BArr, A. y SERra, D. (2009). The effects of externalities and framing on bribery in a petty corruption experiment. Experimental Economics, 12, 488-503.

Berninghaus, S. K.; Haller, S.; Krüger, T.; NeuMAnN, T.; Schosser, S. y Vogt, B. (2013). Risk attitude, beliefs, and information in a corruption 
Game - An experimental analysis. Journal of Economic Psychology, 34, 46-60.

Bertin, J. M. (2010). Fighting corruption: Should we rely on altruistic punishment? Working Paper Department of Social Science.

Beu, D. S.; Buckley, M. R. y Harvey, M. G. (2003). Ethical decision-making: A Multidimensional Construct. Business Ethics, 12 (1), 88-107.

Binmore, K. y Klemperer, P. (2002). The Biggest Auction Ever: The Sale of the British 3G Telecom Licences. The Economic Journal, 112 (478), C74-C96.

Bobkova, N. y Egbert, H. (2012). Corruption Investigated in the Lab: A Survey of the Experimental Literature. International Journal of Latest Trends in Finance and Economic Sciences, 2 (4), 337-349.

Boenm, F. y Lambsdorff, J. G. (2009). Corrupción y anticorrupción: una perspectiva neoinstitucional. Revista de Economía Institucional, 11 (21), 45-72.

Brañas Garza, P. (ed.) (2011). Economía experimental $y$ de comportamiento. Barcelona: Antoni Bosch

Büchner, S.; Freytag, A.; González, L. G. y Güth, W. (2008). Bribery and Public procurement: An experimental study. Public Choice, 137 (1/2), 103-117.

Burke, R. J. (2009). Greed. En Burke, R. J. y Cooper, C. L. (eds.). Research companion to corruption in organizations (pp. 33-59). Northampton: Edward Elgar.

Camerer, C. (2003). Behavioral Game Theory: Experiments in Strategic Interaction (Roundtable Series in Behavioral Economics). Princeton: Princeton University Press.

Campos-Ortiz, F. (2011). Experience, Attitudes and Corrupt Behavior: Insights from an Experiment on Bribery. Working Paper Department of Economics.
DeCelles, K. A.; DeRue, D. S., Margolis, J. D., Joshua, D. y Ceranic, T. (2012). Does Power Corrupt or Enable? When and Why Power Facilitates Self-Interested Behavior. Journal of Applied Psychology, 97 (3), 681-689.

De Fine Licht, J.; Naurin, D.; Esaiasson, P. y GiLLJAM, M. (2014). When Does Transparency Generate Legitimacy? Experimenting on a Context-Bound Relationship. Governance: An International Journal of Policy, Administration, and Institutions, 27 (1), 111-134.

Drugov, M.; Hamman, J. y Serra, D. (2014). Intermediaries in Corruption: An Experiment. Experimental Economics, 17, 78-99.

Druckman, J. N.; Green, P. D.; Kuklinski, H. J. y Lupia, A. (2006). The Growth and Development of Experimental Research in Political Science. American Political Science Review, 100 (4), 627-36.

Dunning, T. (2012). Natural Experiments in the Social Sciences: A Design-Based Approach (Strategies for Social Inquiry). Cambridge: Cambridge University Press

Dusek, L.; Ortmann, A. y Lízal, L. (2005). Understanding Corruption and Corruptibility through Experiments. Prague Economic Papers, 2, 147-162.

Frank, B. y Schulze, G. G. (2000). Does economics make citizens corrupt? Journal of Economic Organization \& Behavior, 43, 101-113.

Frank, B.; Lambsdorff, J. G. y Boehm, F. (2011). Gender and Corruption: Lessons from Laboratory Corruption Experiments. European Journal of Development Research, 23 (1), 59-71.

Frank, R. H., Gilovich, T. D. y Regan, D. T. (1996). Do Economists Make Bad Citizens? The Journal of Economic Perspectives, 10 (1), 187-192.

Feddersen, T.; Gailmard, S. y Sandroni, A. (2009). Moral bias in large elections: Theory and Ex- 
perimental evidence. American Political Science Review, 103 (2), 175-192.

Fink, H. y Boenm, F. (2011). Corrupción en la policía de tránsito. Una primera aproximación a través de entrevistas con taxistas colombianos. Revista Relaciones, 32 (126), 67-85.

Friedman, D. y Cassar, A. (eds.) (2004). Economics Lab. An intensive course in experimental economics. London y New York: Routledge.

Gino, F. y Ariely, D. (2012). The dark side of creativity: Original thinkers can be more dishonest. Journal of Personality and Social Psychology, 102 (3), 445-459.

Gino, F. y Bazerman, M. H. (2009). When misconduct goes unnoticed: The acceptability of gradual erosion in others' unethical behavior. Journal of Experimental Social Psychology, 45 (4), 708-719.

Gino, F.; Ayal, S. y Ariely, D. (2009). Contagion and Differentiation in Unethical Behavior. Psychological Science, 20 (3), 393-398.

Gino, F.; Schweitzer, M. E.; Mead, N. L. y Ariely, D. (2011). Unable to resist temptation: Hoe solfcontrol depletion promotes unethical behavior. Organizational Behavior and Human Decision Processes, 115 (2), 191-203.

Gintis, H. (2009). The Bounds of Reason. Game Theory and the unification of the bahvioral sciences. Princeton: Princeton University Press.

González, A. C. y Boenm, F. (2013). Medir la corrupción: de la generación de conciencia a los peligros de la mala interpretación. Revista Ópera, 11, 259-277.

Guala, F. (2002). On the scope of experiments in economics: Comments on Siakantaris. Cambridge Journal of Economics, 26 (2), 261-267.

Heine, S. J.; Takemoto, T.; Moskalenko, S.; Lasaleta, J. y Henrich, J. (2008). Mirrors in the Head: Cultural Variation in Objective Self-Awareness.
Personality and Social Psychology Bulletin, 34 (7), 879-887.

Hertwig, R. y Ortmann, A. (2008). Deception in Experiments: Revisiting the Arguments in its Defense. Ethics and Behaviour, 18 (1), 59-92.

Hulbert, L. (2004). Experimental Research Designs. En Breakwell, G. M. (ed.). Doing Social Psychology Research. Oxford: BPS Blackwell.

IsAZA, C. (2011). El fracaso de la lucha anticorrupción en Colombia. Revista Ópera, 11, 221-239.

Kish-Gephart, J. J.; Harrison, D. A. y Treviño, L. K. (2010). Bad apples, bad cases, and bad barrels: Meta-Analytical Evidence about Sources of Unethical Decisions at Work. Journal of Applied Psychology, 95 (1), 1-31.

LAMBSDORFF, J. G. (2012). Behavioral and experimental economics as a guidance to anticorruption. En WANTchekon, D. y Wantchekon, L. (eds.). Advances in Experimental Research on Corruption. London: Emerald Books.

Lambsdorff, J. G. y Frank, B. (2010). Bribing versus gift-giving - An experiment. Journal of Economic Psychology, 31 (3), 347-357.

Lambsdorff, J. G. y Frank, B. (2011). Corrupt reciprocity - Experimental evidence on a men's game. International Review of Law and Economics, 31 (2), 116-125.

Liljenquist, K.; Chen-Bo Zhong, C-B. y Galinsky, A. D. (2010). The Smell of Virtue: Clean Scents Promote Reciprocity and Charity. Psychological Science, 21 (3), 381-383.

Mazar, N. y Ariely, D. (2006). Dishonesty in everyday life and its policy implications. Journal of Public Policy \& Marketing, 25 (1), 1-21.

Mazar, N.; Amir, O. y Ariely, D. (2008). The dishonesty of honest people: A theory of self-concept maintenance. Journal of Marketing Research, 45, 633-644. 
Misas, G. (2005). La lucha anticorrupción en Colombia. Bogotá: Contraloría General de la Nación.

Moro, R. y Freidin, E. (2012). Estudios experimentales sobre corrupción y el problema de la validez externa. Interdisciplinaria, 29 (2), 223-233.

Olken, B. (2007). Monitoring Corruption: Evidence from a Field Experiment in Indonesia. Journal of Political Economy, 115 (2), 200-249.

Peisakhin, L. y Pinto, P. (2010). Is transparency an effective anti-corruption strategy? Evidence from a field experiment in India. Regulation \& Governance, 4, 261-280.

Rey-Biel, P. (2008). Economía experimental y teoría de juegos. En Aguilar, F., Barragán, J. y Lara, N. (eds). Economía, sociedad y teoría de juegos (pp. 137-151). Madrid: McGrawHill.

Ryvkin, D. y Serra, D. (2013). Does competition among public officials reduce corruption? An experiment. Working Paper Department of Economics.

Rothstein, B. y Eek, D. (2009). Political Corruption and Social Trust: An Experimental Approach. Rationality and Society, 21 (1), 81-112.

Schikora, J. (2010). Four Essays on Corruption and Cooperation. Theory and Evidence. München: Ludwig-Maximilians-Universität.
Schulze, G. G. y Frank, B. (2003). Deterrence versus intrinsic motivation: Experimental evidence on the determinants of corruptibility. Economics of Governance, 4, 143-160.

Serra, D. y Wantchekon, L. (eds.) (2012). Advances in Experimental Research on Corruption. London: Emerald Books.

Stoker, G. (2010). Exploring the Promise of Experimentation in Political Science: Micro-Foundational Insights and Policy Relevance. Political Studies, 58 (2), 300-319.

Tversky, A. y Kahneman, D. (1986). Rational choice and the framing of decisions. The Journal of Business, 59 (4/2), S251-S278.

Treviño, L. K.; Weaver, G. R. y Reynolds, S. J. (2006). Behavioral Ethics in Organizations: A Review. Journal of Management, 32 (6), 951-990.

Veldhuizen, R. (2013). The influence of wages on public officials' corruptibility: A laboratory investigation. Journal of Economic Psychology, 39, 341-356.

Zhong, C-B. y LiLjenquist, K. (2006). Washing away your sins: threatened morality and physical cleansing. Science, 313 (5792), 1451-2. 